\title{
Optical Limiting Based on Huygens Metasurfaces
}

\author{
Austin Howes ${ }^{1 \dagger}$, Zhihua Zhu ${ }^{2 \dagger}$, David Curie ${ }^{1}$, Jason R. Avila ${ }^{3}$, Virginia D. Wheeler ${ }^{3}$, Richard F. \\ Haglund $^{1}$, and Jason G. Valentine $e^{4 *}$
}

${ }^{1}$ Department of Physics and Astronomy, Vanderbilt University, Nashville, TN 37212, USA

${ }^{2}$ Department of Electrical Engineering and Computer Science, Vanderbilt University, Nashville, TN 37212, USA

${ }^{3}$ U.S. Naval Research Laboratory, Washington, DC 20375, USA

${ }^{4}$ Department of Mechanical Engineering, Vanderbilt University, Nashville, TN 37212, USA

†These authors contributed equally to the work

*Corresponding author: jason.g.valentine@vanderbilt.edu

\section{SUPPLEMENTARY INFORMATION:}

Table 1: Thermal properties of materials in the Huygens metasurface-based optical limiter design.

\begin{tabular}{cccc}
\hline Material & Density $\left(\mathrm{g} / \mathrm{cm}^{3}\right)$ & Thermal Cond. W/K $\cdot \mathrm{m}$ & Heat Capacity $(\mathrm{kJ} / \mathrm{K} \cdot \mathrm{kg})$ \\
\hline $\mathrm{Si}$ & 2.328 & 1.5 & 0.71 \\
$\mathrm{SiO}_{2}$ & 2.4 & 1.4 & 0.75 \\
$\mathrm{VO}_{2}\left(25^{\circ} \mathrm{C}\right)$ & 4.57 & 3.5 & 0.656 \\
$\mathrm{VO}_{2}\left(85^{\circ} \mathrm{C}\right)$ & 4.64 & 6 & 0.78 \\
\hline
\end{tabular}

